Rev. Elev. Méd. Vét Pays trap., 1968, 21, 3 (335-37).

\title{
Essais de traitement de la péripneumonie contagieuse bovine par la mépacrine
}

\author{
por A. PROVOST ef R. QUEVAL \\ (I. E. M. V. T., Laboratoire de Recherches Vétérinaires de Farcha, Fort-Lamy, Tchad)
}

\begin{abstract}
RÉSUMÉ
Bactériostatique in vitro vis-à-vis de $M$. mycoides à la quantité de $10 \mu \mathrm{g} / \mathrm{ml}$, la Mépacrine (Quinacrine Spécia) n'arrête pourtant pas in vivo le déroulement du phénomène de Willems ni ne guérıt, même cliniquement, la péripneumonie déclarée.
\end{abstract}

En Afrique intertropicale, ce n'est pas dans les grands effectıfs bovins pastoraux que la péripneumonie est redoutée i son incidence $y$ est d'ailleurs faible. C'est dans les zones d'élevage sédentaire, chez des agriculteurs possédant une ou deux paires de boufs, que la reconnaissance de la maladie pose des problèmes souvent insolubles. La logique sanitaire voudrait qu'on y procédât à l'abattage dont la pratique - l'un de nous l'a mantré ailleurs (4) — n'est pas actuellement compatible avec les mours ou l'état d'évolution des populations. Aussi, malgré les objections qu'y opposent à juste trtre hygiénistes et planificateurs, certains praticiens recherchent toujours un médicament bon marché qui, sauvant de la mort les bovins péripneumoniques, permettrait de les diriger à plus ou moins bref délai vers la boucherie. Dans cette optique, ef avec ces limites, le novarsénobenzol a conservé de chauds partisans (3).

II n'est pourtant pas sans désavantages. SI l'on excepte le principol, celui qui le condamne et qui est la quası-certıtude de création de porteurs chroniques de lésions encapsulées, il lui reste encore à surmonter les handicaps du prix d'achat et surtout de la nécessité d'intervention d'un personnel qualifié apje à procéder aux inoculations intraveineuses, de surcroît différées dans le temps sur une dizaine de jours.

If conviendrait donc pour un éventuel médica- ment de la péripneumonie qu'il fôt bon marché, stable et capable d'être administré par l'éleveur lui-même. Cette conclusion est autant valable pour le traitement de la streptothricose que celuı des helminthiases.

C'est ayant en mémoire ces impératifs que nous avons pris connaissance avec intérêt du travail de VAN DEMARK (6) qui montre que les flavoprotéınes interviennent dans le cycle respiratoire des mycoplasmes à la place du système hémique des cellules aérobies.

Or il est bien connu en chimie biologıque que l'oxydation des lactates par les flavines est entravée par des inhibiteurs tels que certains barbituriques ou encore l'atébrine.

Ce dernier corps est un produit de la grande synthèse pharmaceutique, étant le dichlorhydrate de méthoxy-2 chloro-6 (diéthylamino-4' méthyl-1') -butyl) amino-9 acridine (*), largement utilisé en son temps comme antipaludéen mass maintenant réservé au traitement des coccidioses anımales. Si in vitro comme in vivo ce corps se montrait actıf, il serait assuré de quelque succès car répondant aux désirs exprimés, notamment parce qu'il est administrable per os.

(*) Cammercialisé en France par Spécıa sous le nom de Quinacrine. II nous est agréable de remercier cette firme en la personne de notre confrère le Docteur FERRIOT, qui a aimablement mis des échantillons à notre disposition 
Les essais auxquels il a conduit sont rapportés dans cette note.

\section{MATÉRIEL ET MÉTHODES}

\section{A. - Essais in vifro.}

Ils consistent à rechercher classiquement la plus petite quantité de mépacrine ayant un effet bactériostatique sur une culture d'une souche pathogène de Mycoplasma mycordes.

La souche utilisée à cet effet est «Afadé», souche pathogène près de son isolement à partir d'un poumon péripneumonique, cultivée en milieu F-66 (2). Des quantités de mépacrine allant de $10 \mathrm{mg}$ dे $0,00001 \mathrm{ug} / \mathrm{ml}$ sont réparties dans des tubes de milieu $\mathrm{F}-66$ qui sont ensuite ensemencés avec $0,5 \mathrm{ml}$ d'une culture de 48 heures de la souche péripneumonique. La lecture intervient après séjour de 7 jours d̀ l'étuve à $37^{\circ} \mathrm{C}$; dans les tubes où n'existe apparemment aucune culture, l'absence de développement des mycoplasmes est contrôlée par observation en microscopie de phase.

\section{B. - Essais in vivo.}

On a recherché l'effet curatif de la mépacrine sur le phénomène de WILLENS et la péripneumonie expérimentale, cette dernière en tous points analogue à la maladie naturelle.

1. Souche. La même souche Afadé a été utilisée sous forme de broyat de poumon péripneumonique d̀ 20 p. 100 en milieu F-66, pour réaliser des intubations intrabronchiques de malades «donneurs» de péripneumonie et a été, en d'autres occasions, employée pour produire des phénomènes de Willems ; une souche, baptisée C 11, isolée d'un poumon de chèvre, a servi dans le même but.

2. Bovins. Animaux de race arabe choa, âgés de 2 ans environ, non vaccinés contre la péripneumonie. La péripneumonie expérimentale est produite chez certains d'entre eux en les plaçant dans un enclos au contact de bovins ayant reçu une intubation endobronchique de broyat de poumon péripneumonique. Le traitement commence à des délais variables après le contact mais toujours dès l'expression clinique de la maladie (jetage nasal, toux, essoufflement d'effort).
Le phénomène de WILLEMS est, quant à lui, très aisément déclenché par la sımple inoculation sous-cutanée rétro-scapulaire de cultures des souches Afadé et C 11. On commence l'administration de mépacrıne aussitôt que devient palpable l'œdème réactionnel.

3. Tests sérologiques. Le retentissement sérologique des deux maladies expérimentales est suivi par des réactions de fixation du complément : test dit de FARCHA (2) et test des quatre tubes (5).

4. Conduite de lo thérapeutique. Tenant compte du résultat des essais de bactériostase in vitro, la posologie établie a été de $250 \mathrm{mg}$ par jour et par kilo de poids vif, chiffre qui ne s'écarte pas de ce qui est prescrit par le fabricant pour le traitement de la coccidiose bovine (1). La dose quotidienne est répartie en deux prises égales et données à la bouteille après dissolution dans de l'eau du robinet (1 litre d'eau pour $2 \mathrm{~g}$ à $2,5 \mathrm{~g}$ de produit), Le traitement est institué pendant 5 jours en principe : ll y a dû être écourté en quelques circonstances devant l'évolution de la maladie.

\section{RÉSULTATS}

\section{A. - Essais in vitro.}

La mépacrine est bactériostatique d̀ la quantıté de $10 \mu \mathrm{g} / \mathrm{ml}$ de mılieu F-66. C'est une activité qul est voisine de celle des antibiotiques sur les mycoplasmes et qui donc autorise des espoirs thérapeutiques.

\section{B. - Essais in vivo.}

Sur 9 bovins traités, un seul a vu régresser ses symptômes et a guéri cliniquement. II a néanmoins conservé la trace sérique de son infection chronique par des décharges d'antigène péripneumonique circulant (bovin no 3907 du tableau 4 de la référence 5).

Les autres sont morts de péripneumonie ou ont été sacrifiés agonisants. $M$. mycoides est très aisément isolé des lésions pleuro-pulmonaires.

Les sept bovins présentant des réactions willemsiennes (4 avec la souche Afadé, 3 avec la souche $\mathrm{C}$ 11) ne répondent pas au traitement et sont sacrifiés à l'agonie. 


\section{DISCUSSION}

Comme pour beaucoup d'expériences, on pourrait conclure qu'il y a loin de la coupe aux lèvres. La mépacrine administrée per os ne justifie pas les espoirs nés de l'observation de son activité bactériostatique in vitro sur $M$. mycoïdes.

Le seul point positif est qu'à la posologie relativement élevée utilisée, elle se montre dénuée de tout effet clinique fâcheux.

Cef échec ne s'explique pas par une insensibilité particulière de la souche utilisée pour conférer la maladie expérimentale ; c'est la même que celle ayant servi aux essais en tubes. II n'y a pas lieu d'épiloguer ici sur le mécanisme de la résistance, soulignant simplement qu'il est possible que dans les humeurs du bovin $M$. mycoides trouve une autre chaîne respiratoire suppléante ou encore que la mépacrine soit liée et de ce fait partiellement ou totalement inactivée ; cette dernière explication n'est au demeurant guère satisfaisante au regard de son succès thérapeutique dans les coccidioses.

Il reste au total que le médicament actif, bon marché et d'emploi aisé contre la péripneumonie bovine reste à découvrir. Compilant les connaissances que l'on a sur la pathogénie de la maladie et gardant en mémoire la facilité qu'ont les bovins d'encapsuler et de séquestrer des lésions inflammatoires du poumon ou d'autres parenchymes, on doit se demander si pour l'instant tout traitement stérilisant ne relève pas de I'utopie, Tout autre, on l'a vu, est pourtant l'optique que l'on en a dans certains pays ; la conclusion est donc en définitive qu'il faut persévérer dans la recherche d'un médicament efficace.

\section{SUMMARY}

Therapeutical trials with « mepacrine » against contagious bovine pleuropneumonla

"Mepacrine 》 (Quinacrine Spécia) is bacteriostatic in vitro in regard to M. mycoides at dosis of $10 \mu \mathrm{g} / \mathrm{ml}$. Yet it does not stop in vivo the process of development of Willems's réaction nor cure clinical pleuro-pneumonia.

\section{RESUMEN}

Ensayos de tratamiento de la perineumonia contagiosa de los bovinos por la « mepacrine»

La mepacrine (Quinacrine Spécia) es bacteriostática in vitro para con M. mycoides en la dosis de $10 \mu \mathrm{g} / \mathrm{ml}$. Sin embargo no detiene in vivo el proceso del fenómeno de Willems y no cura, hasta clinicamente, la perineumonia.

\section{RÉFÉRENCES}

1. ANONYME. - Thérapeutique vétérinaire. Nomenclature générale des produits Spécia. $10^{\mathrm{e}}$ éd. Spécia, (21, rue Jean-Goujon Paris 8e) 1963-1964.

2. ANONYME. - Rapport annuel de la Région de Recherches Vétérinaires et Zootechniques d'Afrique centrale pour l'année 1966. Tome 2 : Rapport quinquennal sur la péripneumonie.

3. ORUE (J.) et MEMERY (G.). - Lo péripneumonie bovine. Traitement par le novarsénobenzol. Rev. Elev. Méd. vét. Pays trop., 1961, $14: 405-411$.
4. PROVOST (A.). - Etat actuel de l'immunisalion active dans la péripneumonie bovine. A paraître.

5. PROVOST (A.) et QUEVAL. (R.). - Recherches immunologiques sur la péripneumonie. $X$. Proposition d'une nouvelle technique de diagnostic expérimental de la maladie : le test des quatre tubes. Rev. Elev. Méd. vét. Pays trop., 1968, 21.

6. VAN DEMARK (P. J.). - Respiratory pathways in the mycoplasmas. Ann. N. Y. Acad. Sc., 1967, $143:$ 77-84. 